\section{Silicon-based quantum wells}

SIR - Since our proposal ${ }^{l}$ and subsequent observation $^{2}$ of resonant tunnelling in quantum-well structures, the field has expanded to include all kinds of quantum ${ }^{3}$ and functional devices ${ }^{4}$. As long as the electron mean-free-path is greater than the dimensions of the quantum well, pseudo-eigenstates can form in the well. To date, the wells and barriers are formed with lattice-matched systems such as

based quantum well.

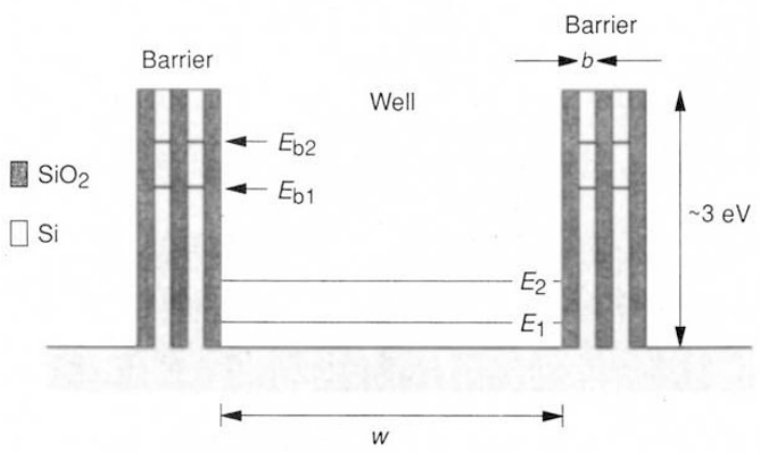

A Si/SiO ${ }_{2}$ quantum well using a $\mathrm{Si} / \mathrm{SiO}_{2}$ superlattice as the barrier.

GaAs/AlGaAs, GaInAs/AlInAs and so on. Because the electronics industry is overwhelmingly dominated by silicon integrated-circuit processing technology, these new devices cannot play an economic technological role unless quantum confinement in silicon can be achieved with barriers much higher than those possible with $\mathrm{Si} / \mathrm{Si}_{1-x} \mathrm{Ge}_{x}$ systems ${ }^{5}$. Without a higher barrier, it is impossible to create a device operating at a voltage corresponding to an energy much above $k_{\mathrm{B}} T$ at room temperatures. Because of the lack of a suitable material for barriers, I propose that strain-layer superlattices ${ }^{6-8}$ be used as barriers.

Basically, the concept of a strain-layer superlattice is that, with a sufficiently thin epitaxial layer, the strain energy in each layer is below the energy needed for the growth of point defects or dislocations. It is important to note that dislocations have an activation energy for nucleation and a lower activation energy for growth. Therefore, in principle it is possible greatly to exceed the energy requirement without actually generating defects.

The MOS device owes its success to the low defect density at the Si/amorphous$\mathrm{SiO}_{2}$ interface. With a barrier height of 3.2 eV, amorphous $\mathrm{SiO}_{2}$ should be an ideal barrier for quantum confinement in silicon. Unfortunately, it is not possible to grow epitaxial silicon on amorphous $\mathrm{SiO}_{2}$. But if the $\mathrm{SiO}_{2}$ layer is very thin (a couple of monolayers) it remains crystalline, so it should then be possible to grow on top of it an epi-layer of silicon. This is the basis for my proposal that a strain-layer superlattice be used as the barrier for a silicon-
It is often assumed in strain-layer superlattices that a large lattice mismatch results in high defect density which destroys quantum confinement. But in fact, not only is scattering less effective in lower-dimensional systems, but it fails to destroy phase coherence in zero-dimensional systems such as quantum dots if the scattering process possesses timereversal symmetry ${ }^{9}$. Consequently, in an isolated system such as a quantum well, defects may not be detrimental.

A strain-layer epitaxial $\mathrm{Si} / \mathrm{SiO}_{2}$ barrier system is shown in the figure. Because the width $b<<w, E_{\mathrm{b} 1}$ and $E_{\mathrm{b} 2}$ are much higher than $E_{1}$ and $E_{2}$, the quantum well states. This is the basis of a strain-layer barrier. The effective barrier height may be as high as $3 \mathrm{eV}$, so it is possible to design the separation $E_{2}-E_{1}$, depending on the width $w$, to be much greater than $k_{\mathrm{B}} T$ at room temperature. If, for example, one can generate four states with separations of $\sim 0.5 \mathrm{eV}$, a four-level multiple logic circuit operating at steps of $0.5 \mathrm{~V}$ could be designed.

Other materials for our scheme could be the $\mathrm{Si} / \mathrm{Al}_{2} \mathrm{O}_{3}$ system, which has been known for many years, or the more recent $\mathrm{Si} / \mathrm{CaF}_{2}$ system ${ }^{10}$. My choice is the $\mathrm{Si} / \mathrm{SiO}_{2}$ system because $\mathrm{SiO}_{2}$ can be grown by exposing a silicon substrate heated to $\sim 550{ }^{\circ} \mathrm{C}$ to oxygen. Subsequently, silicon can be evaporated or even deposited epitaxially with a molecular source. Hydrogen implantation followed by moderate annealing could be used to provide hydrogen passivation of possible dangling bonds. Therefore it may be better to deposit the $\mathrm{SiO}_{2}$ onto a silicon surface oriented several degrees off any high symmetry axis, such as the $(100)$, to avoid nucleation of defects at the steps.

Finally, to take advantage of small devices (low RC discharge time), it is desirable further to confine the electrons into a dot. Ultimately, the full use of such a quantum-well device will rely on nanoscale lithography.

\section{Raphael Tsu}

Department of Electrical Engineering, University of North Carolina,

\section{Charlotte, North Carolina}

and

Nanodynamics, Inc.,

510 E 73rd Street, New York,

New York 10021, USA

\footnotetext{
1. Tsu, R. \& Esaki, L. Appl. phys. Lett. 22, 562-564 (1973)

2. Chang, L. L., Esaki, L. \& Tsu, R. Appl. phys. Lett. 24 593-595 (1974).

3. Capasso, F. Physics of Quantum Devices, 181-248 (Springer, Berlin, 1990).

4. Capasso, F. \& Kiehl, R. H. J. appl. Phys. 58, 1366-1368 (1985).

5. Chen, Y. F. etal. Appl. phys. Lett. 62, 2713-2715 (1993)

6. Tsu, R. US Patent No. 5216262 (1993).

7. Matthews, J. W. \& Blakeslee, A. E. J. cryst. Growth 32 265-273(1976).

8. Osbourn, G. C.J. appl Phys, 53, 1586-1589 (1982).

9. Tsu, R. Physica $B$ (in the press)

10. Sinharoy, S., Greggi, J. \& Schmidt, D. N. J. appl. Phys. 64, 6296-6300 (1988)
}

\section{Database of ancient sequences}

SIR - Green et al. ${ }^{1}$ have introduced the concept of ancient conserved regions (ACRs), defined as contiguous aminoacid sequence segments predating the coelomate radiation $500-600$ million years ago. Statistical arguments strongly suggest that currently known proteins of vertebrates, invertebrates, yeast and bacteria may already include representatives of most ACRs. Thus the numerous new protein domains or motifs that will emerge from sequencing yeast or nematode are likely to be restricted to these phyla.

I have estimated the total number and assembled a repertoire of these ancestral sequences from an analysis of the SwissProt (21.0) protein database ${ }^{2}$. First, I have isolated seven comprehensive subsets of protein sequences from eubacteria, plants, fungi, slime mould, vertebrates,
CROSS-PHYLUM COMPARISONS OF SELECTED SEQUENCE SUBSETS

\begin{tabular}{|c|c|c|c|c|c|c|}
\hline \multirow[b]{2}{*}{ Subsets } & \multirow[b]{2}{*}{ Entries } & \multirow{2}{*}{$\begin{array}{l}\text { Target } \\
\text { entries }\end{array}$} & \multicolumn{2}{|c|}{ Score $>84\left(P=5 \times 10^{-5}\right)$} & \multicolumn{2}{|c|}{ Score $>69\left(P=2.5 \times 10^{-2}\right.$} \\
\hline & & & $\begin{array}{l}\text { Matching } \\
\text { other phyla }\end{array}$ & ACRs & $\begin{array}{c}\text { Matching } \\
\text { other phyla }\end{array}$ & ACRs \\
\hline Vertebrates & 6,578 & 6,410 & $2,323(35 \%)$ & 390 & $2,605(40 \%)$ & 374 \\
\hline Bacteria & 2,543 & 10,445 & $521(20 \%)$ & 281 & $602(24 \%)$ & 280 \\
\hline Plants & 2,224 & 10,762 & $860(39 \%)$ & 199 & $975(44 \%)$ & 210 \\
\hline Yeasts & 984 & 12,004 & $561(57 \%)$ & 303 & $606(62 \%)$ & 307 \\
\hline Drosophila & 440 & 12,548 & $317(72 \%)$ & 153 & $334(76 \%)$ & 139 \\
\hline Slime mould & 124 & 12,864 & $68(54 \%)$ & 45 & $73(59 \%)$ & 47 \\
\hline Nematode & 94 & 12,894 & $68(72 \%)$ & 45 & $69(73 \%)$ & 40 \\
\hline All & 12,988 & NA & $4718(36 \%)$ & 551 & $5,264(40 \%)$ & 520 \\
\hline
\end{tabular}

The final set of ACR representatives is obtained by comparing the ACRs defined from each subset, forming clusters of related sequences and retaining a single sequence by similarity cluster. Although the number of cross-phylum matches varies with the score threshold (see figure), the final number of independent ACR representatives remains quite stable. Matches were scored according to the optimal pam120 matrix. Change in the scoring matrix did not significantly alter those results. 